\title{
Insights To Emitter Saturation Current Densities Of Boron Implanted Samples Based On Defects Simulations
}

\author{
K. R. C. Mok ${ }^{1}$, R. C. G. Naber ${ }^{2}$ and L. K. Nanver ${ }^{1}$ \\ ${ }^{I}$ Dept. Microelectronics, Delft University of Technology, Feldmannweg 17, 2628 CT Delft, The Netherlands, \\ ${ }^{2}$ Tempress Systems BV, Radeweg 31, 8171MD Vaassen, The Netherlands
}

\begin{abstract}
Emitter saturation current densities, $\mathrm{J}_{\mathrm{oe}}$ have been investigated with different boron implantation dose and annealing conditions. The higher thermal budgets used here are shown experimentally to improve $\mathrm{J}_{\text {oe }}$, implying more complete defect dissolution. Simulations show that significant degradation in $\mathrm{J}_{\mathrm{oe}}$ can be attributed to the presence of dislocation loops. In addition, in cases where dislocation loops have been annealed, high dose boron implantation still results in stable boron interstitial clusters, which contributes to $\mathrm{J}_{\mathrm{oe}}$ degradation.
\end{abstract}

Keywords: Ion implantation, Emitter saturation current density, dislocation loops, boron interstitial clusters PACS: $85.40 . \mathrm{Ry}, 88.40 . \mathrm{hj}$

\section{INTRODUCTION}

Doping in the photovoltaic industry is most commonly realized by furnace diffusion. For the formation of n-type emitter on a p-type base, phosphorus oxychloride $\mathrm{POCl}_{3}$ is usually used. Gaseous $\mathrm{POCl}_{3}$ mixed with oxygen, deposits phosphorus oxide $\mathrm{P}_{2} \mathrm{O}_{5}$ directly onto the wafer surface. Phosphorus then diffuses into the silicon at the furnace temperature $\left(\sim 900^{\circ} \mathrm{C}\right)$. Alternatively, boron tribromide $\mathrm{BBr}_{3}$ can be used as a source for the formation of $\mathrm{p}$ type emitter on an n-type base. In both cases, phosphorus- or boron-doped glass on the wafer surface has to be subsequently etched off using HF.

Recently, the ion implantation doping technique that is widespread in the integrated circuit (IC) industry has emerged as a promising alternative in solar cell production. In general, compared to furnace diffusion, ion implantation offers improved control, uniformity and reproducibility over the dopant profile; and the elimination of the wet processing steps necessary for the etching of phosphorus- or borondoped glass. Moreover, directional, localized, onesided doping can be achieved in a single step using a shadow mask. This comparative advantage allows for novel process flows and high-efficiency cell architecture, for example in the interdigitated back contact solar cell [1].

However, the use of ion implantation is still not dominant due to fundamental requirements of the photovoltaic industry in terms of throughput $(\sim 1500$ wafers per hour) and cost. In addition, the critical requirements for solar cells are a defect-free, wellpassivated substrate that enables high carrier lifetimes; and sufficient dopant activation for good conduction and contacting properties. This is the main technological concern, as it is well-known that ion implantation induces defects that may not be completely removed in subsequent annealing, and may additionally induce dopant deactivation.

Even at low energy and ion doses, ion implantation introduces defects into the silicon lattice. Upon annealing, interstitials and vacancies recombine, leaving excess interstitials. These can evolve from small interstitial clusters to extended $\{311\}$ rod-like defects, which are unstable above $700^{\circ} \mathrm{C}$. With sufficient damage dose, these $\{311\}$ defects then evolve into dislocation loops, which are stable up to $1000^{\circ} \mathrm{C}$. During defect evolution, interstitial point defects released from the extended defects may result in transient enhanced diffusion of dopants and dopant deactivation. The defects are finally annihilated when they diffuse to the surface or interface.

Regardless of the concern over defects, the use of ion implantation [1-6] and annealing [7] applied to solar cells has been studied for many years. Lifetime degradation due to ion implantation induced defects has been explained in terms of near-surface stable dislocation loops, as well as deeply-penetrating defects that result in uniformly-degraded lifetimes up to a depth of $100 \mu \mathrm{m}$ [5]. It has been shown that both ion implantation and annealing conditions affect the resultant carrier lifetimes. Higher ion dose resulted in a greater amount of residual damage and hence lower lifetime $[4,6]$. Also, more complete damage annealing at higher temperature results in a longer carrier lifetime. Even for boron implantations that do not result in extended defects, lifetime degradation was attributed to stable boron-interstitial clusters which remain after a $1000^{\circ} \mathrm{C} 10 \mathrm{~min}$ anneal [6]. 
In this work, the effect of boron ion implantation parameters and annealing process conditions on emitter saturation current densities is investigated. In addition, simulations were performed to gain insights into residual defects, revealing both dislocation loops and boron interstitial clusters, and their effect on emitter saturation current densities.

\section{EXPERIMENTAL PROCEDURES}

In this work, blanket double-side implanted wafers were prepared using 4-inch double-side-polished, float-zone monocrystalline silicon wafers. The wafers are n-type, $300 \mu \mathrm{m}$ thick and have resistivities from 1 to $5 \Omega \mathrm{cm}$. The thermal oxide was first grown for surface passivation before implantation to try to separate the effects of ion implantation induced damage annealing and oxidation. Boron was implanted with a fixed energy of $15 \mathrm{keV}$, a tilt and rotation of $7^{\circ}$ and $22^{\circ}$ respectively to avoid channeling. The implantation doses used were $5 \times 10^{14}, 1 \times 10^{15}$ and $2 \times 10^{15} \mathrm{~cm}^{-2}$. Next, thermal annealing was done in a furnace in nitrogen ambient to avoid any additional oxidation. In order to investigate the effect of various annealing conditions on the dissolution of ion implantation induced damage, anneals were done at $950^{\circ} \mathrm{C}$ for $30 \mathrm{~min}$ and at $1050^{\circ} \mathrm{C}$. In addition, a third anneal condition is included for comparison whereby rapid thermal anneal (RTA) with a peak temperature of $1050^{\circ} \mathrm{C}$ was done in an ASM Epsilon after the $950^{\circ} \mathrm{C}$ furnace anneal. It is worth mentioning that for practical application in solar cell production, the combination of damage annealing and oxidation would be advantageous from a cost point of view.

Finally, the emitter saturation current density $\left(\mathrm{J}_{\mathrm{oe}}\right)$ was measured by the quasi-steady state photoconductance (QSSPC) method [8]. The sheet resistance was measured by 4-point probing.

Modeling and simulation of ion implantation induced damage evolution and dopant diffusion has long been used in the IC industry to develop the required dopant profiles and activation [9]. In this work, simulations were performed using Synopsys' TCAD Sentaurus kinetic Monte Carlo code that includes ion implantation induced damage evolution, as well as dopant-defect clustering phenomena [9-10]. However, the mechanism of carrier lifetime degradation is dependent not only on the recombination centres in the presence of defects, but also on the type of interface between the $\mathrm{Si}$ and the dielectric, and on the collected charge inside the dielectric. The simulation results shown in this work provide a relative comparison of the residual ion implantation damage, and this can be correlated to $\mathrm{J}_{\mathrm{oe}}$ since the passivation layer is the same for all the samples. The effects of passivation layers and dielectric charge are not taken into account in the simulations.

\section{RESULTS AND DISCUSSION}

Figure 1 shows the sheet resistance as a function of implanted dose for samples that were implanted with boron at $15 \mathrm{keV}$, followed by a thermal anneal at $950^{\circ} \mathrm{C}$ for $30 \mathrm{~min}$. Simulation results show that the experimental sheet resistance can be accurately reproduced, giving confidence in the simulated dopant profile and activation. It is worth noting that for industrial screen-printed solar cell, an emitter sheet resistance of about $60 \Omega / \mathrm{sq}$ is commonly used. This requires a high dose implantation of about $2 \times 10^{15} \mathrm{~cm}^{-2}$.

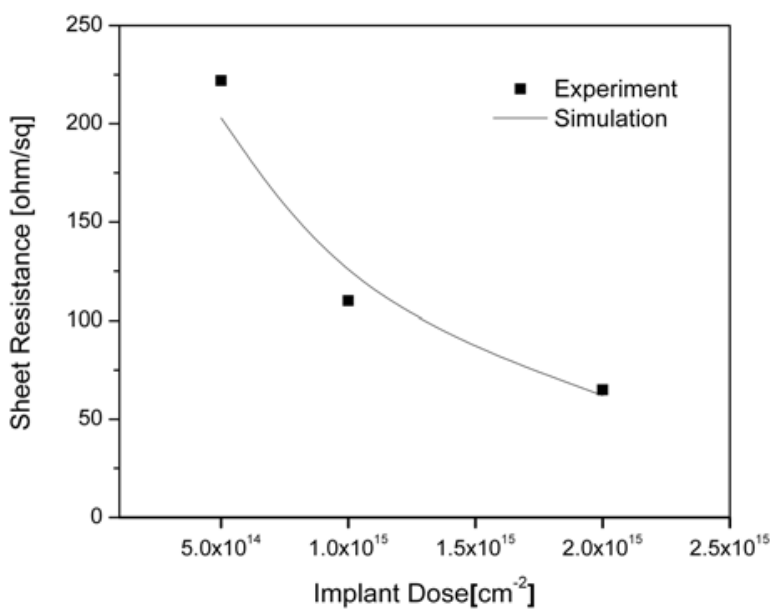

FIGURE 1. Sheet resistance as a function of implanted boron dose at $15 \mathrm{keV}$, followed by thermal annealing at $950^{\circ} \mathrm{C}$ for $30 \mathrm{~min}$.

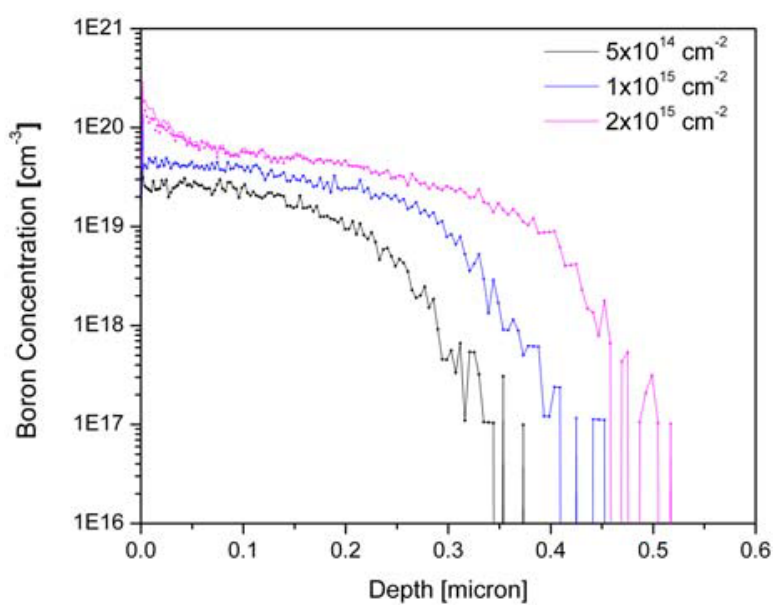

FIGURE 2. Simulated boron concentration profiles at different implanted boron doses at $15 \mathrm{keV}$, followed by thermal annealing at $950^{\circ} \mathrm{C}$ for $30 \mathrm{~min}$. Lines: total boron and symbols: active boron. 
Figure 2 shows the simulated boron concentration at different implanted doses, followed by a $950^{\circ} \mathrm{C} 30$ min thermal anneal. These implantation conditions do not result in an amorphous layer.

Figure 3 shows the $\mathrm{J}_{\mathrm{oe}}$ measured by QSSPC in samples as a function of boron implanted dose. The samples were annealed under different conditions.

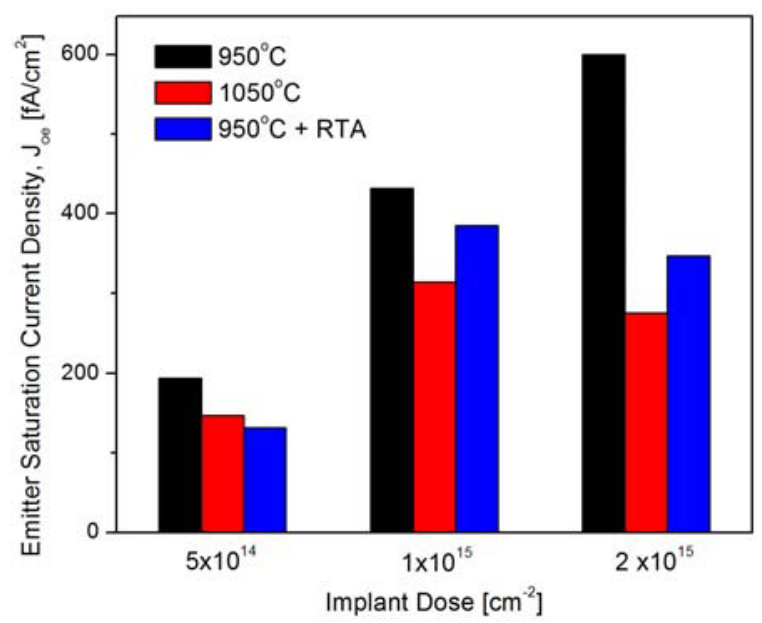

FIGURE 3. Emitter Saturation Current Density, $\mathrm{J}_{\mathrm{oe}}$, as a function of implanted boron dose at $15 \mathrm{keV}$, followed by thermal annealing at $950^{\circ} \mathrm{C}, 1050^{\circ} \mathrm{C}$ and $950^{\circ} \mathrm{C}$ followed by RTA.

For samples that were annealed at $950^{\circ} \mathrm{C}$ for 30 min, $J_{o e}$ increases with implant dose. This is in agreement with results of Pawlak et. al. [6] that show degradation of the effective lifetime in the same dose range following a $10 \mathrm{~min}$ anneal at $1000^{\circ} \mathrm{C}$. Compared to samples annealed at $950^{\circ} \mathrm{C}$, the $\mathrm{J}_{\mathrm{oe}}$ of samples annealed at $1050^{\circ} \mathrm{C}$ and $950^{\circ} \mathrm{C}$ followed by RTA is observed to decrease, corresponding to the fact that a higher thermal budget is more effective at annealing defects. This decrease in $\mathrm{J}_{\mathrm{oe}}$ with increase in thermal budget is marginal for implant doses of $5 \times 10^{14} \mathrm{~cm}^{-2}$ and $1 \times 10^{15} \mathrm{~cm}^{-2}$. However, for the highest dose implant at $2 \times 10^{15} \mathrm{~cm}^{-2}$, a drastic drop in $J_{o e}$ with increase in thermal budget is observed.

Figure 4 shows the simulated residual defect (interstitial) doses for various boron implant doses and anneal conditions. For $5 \times 10^{14} \mathrm{~cm}^{-2}$ implants, no defects (neither extended dislocation loops nor boroninterstitial clusters) remain in the simulations. This can also be seen from Figure 2, where the total boron concentration profile is active for $5 \times 10^{14} \mathrm{~cm}^{-2}$ implant followed by $900^{\circ} \mathrm{C} 30 \mathrm{~min}$ anneal. However, the experimental $\mathrm{J}_{\mathrm{oe}}$ is observed to drop with higher thermal budget anneals even for this low implant dose. This could be due to a more complete annealing of the deeply-penetrating defects, as a result of a higher probability that any diffusing defects reach the wafer surface or interface with higher thermal budget. These deeply-penetrating defects are known to be distributed uniformly down to a depth of at least $100 \mu \mathrm{m}$ [4-5]. However, these defects are not evident in the simulations. Due to the atomistic nature of the kinetic Monte Carlo simulations, the simulation time is proportional to the size of the simulation domain. Therefore, the simulation domain is focused on the near-surface region, where most of the defects and dopant-defect interactions take place

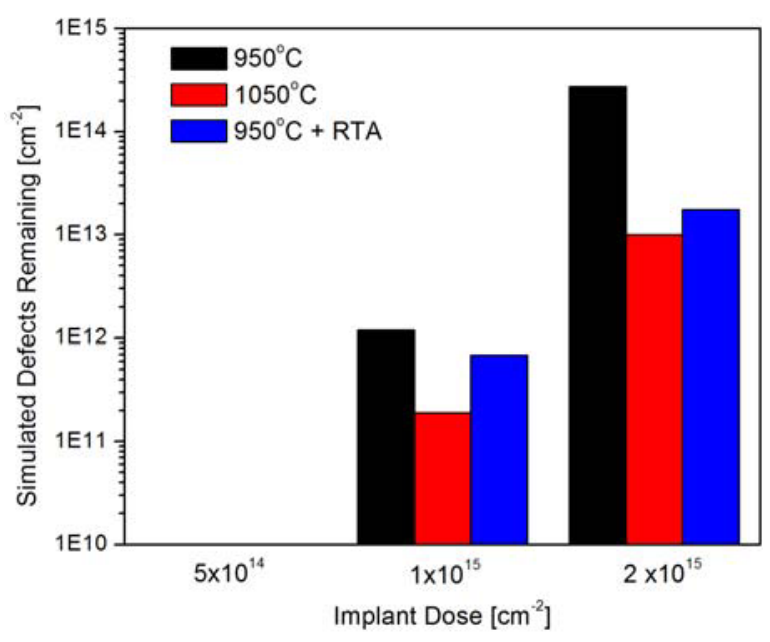

FIGURE 4. Simulated interstitial defects remaining as a function of implanted boron dose at $15 \mathrm{keV}$, followed by thermal annealing at $950^{\circ} \mathrm{C}, 1050^{\circ} \mathrm{C}$ and $950^{\circ} \mathrm{C}$ followed by RTA.

For $1 \times 10^{15} \mathrm{~cm}^{-2}$ implants, simulations show boroninterstitial clusters, predominantly of the $\mathrm{B}_{3} \mathrm{I}$ configuration, remaining at the end of all three anneal conditions. Additional RTA following a $950^{\circ} \mathrm{C}$ anneal resulted in better boron interstitial clusters dissolution, and thereby lower $\mathrm{J}_{\mathrm{oe}}$. For this implant, $1050^{\circ} \mathrm{C}$ furnace anneal proves to be the best annealing condition, with the least amount of residual defects and $\mathrm{J}_{\mathrm{oe}}$. However, simulation shows that some boron interstitial clusters still remain.

Dislocation loops were observed in the simulation for an implant dose of $2 \times 10^{15} \mathrm{~cm}^{-2}$ followed by $950^{\circ} \mathrm{C}$ anneal. In this case, dislocation defects made up $85 \%$ of the total interstitial defects remaining. The rest of the interstitial defects can be attributed to boron interstitial clusters, as can be seen from the difference between total and active boron concentrations in the near surface region in Figure 2. Simulations of an additional RTA or $1050^{\circ} \mathrm{C}$ furnace anneal show no dislocation loops remaining, as well as improved dissolution of the boron interstitial clusters. The drastic drop in $\mathrm{J}_{\mathrm{oe}}$ with higher thermal budget for 
implant dose of $2 \times 10^{15} \mathrm{~cm}^{-2}$ as seen in Figure 3, can be explained from the simulations due to the presence of dislocation loops after a $950^{\circ} \mathrm{C}$ anneal and the dissolution of these dislocation loops after a subsequent RTA or a $1050^{\circ} \mathrm{C}$ furnace anneal.

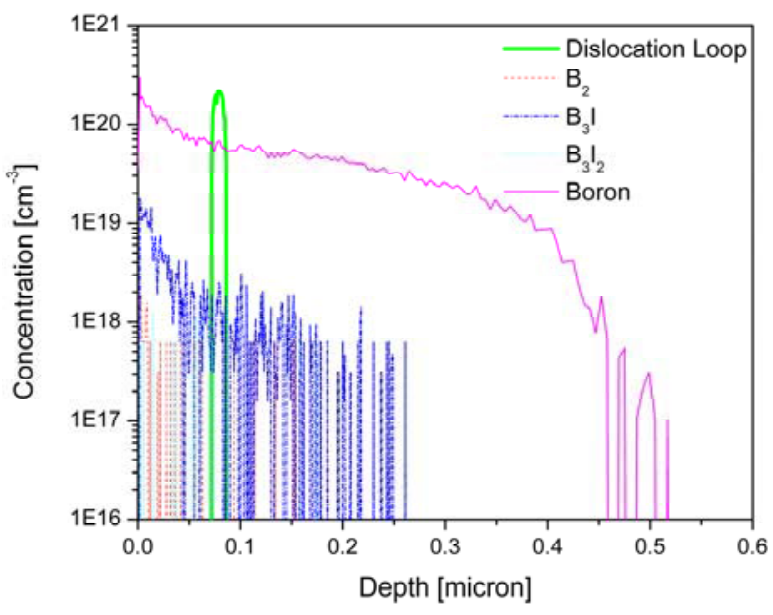

FIGURE 5. Simulated concentration profiles at $2 \times 10^{15} \mathrm{~cm}^{-2}$ implanted boron dose at $15 \mathrm{keV}$, followed by thermal annealing at $950^{\circ} \mathrm{C}$ for $30 \mathrm{~min}$.

Figure 5 shows the simulated concentration profiles of boron and various defects at implanted boron dose of $2 \times 10^{15} \mathrm{~cm}^{-2}$, followed by a $950^{\circ} \mathrm{C} 30 \mathrm{~min}$ thermal anneal. As can be seen, the dislocation loops and boron interstitial cluster defects are located at the nearsurface region. It can be expected that etching and repassivating the surface region would improve the lifetime by removing the defects, consistent with results from Macdonald et al. [4].

The standard boron emitter sheet resistance of about $60 \Omega$ /sq necessitates a high dose boron implant of about $2 \times 10^{15} \mathrm{~cm}^{-2}$. Damage induced by high dose ion implantation is likely to evolve into stable dislocation loops. An anneal condition with insufficient thermal budget would leave behind residual dislocation loops, which significantly degrade $\mathrm{J}_{\mathrm{oe}}$. Even with the complete dissolution of dislocation loops, very stable boron interstitial clusters can still persist to be detrimental to $\mathrm{J}_{\mathrm{oe}}$. Although lifetime and $\mathrm{J}_{\mathrm{oe}}$ depends on defect-free, well-passivated substrate, in the presence of defects, lifetime degradation at high boron ion implant doses has been shown to be identical for both thermal oxide and AlOx, which is known to be a better passivating dielectric [5].

\section{CONCLUSION}

Emitter and back surface field doping in solar cells have distinct process requirements related to implant parameters and annealing thermal budget. Simulations can offer useful insights during process optimization in terms of sheet resistance, dopant profile and the presence of residual defects.

In this work, lifetime degradation and high $\mathrm{J}_{\mathrm{oe}}$ of the experimental results have been correlated to the presence of dislocation loops in simulations. In cases where the implantation dose is below the threshold for nucleation of dislocation loops or where dislocation loops have been annealed, boron implantation above a dose of about $10^{15} \mathrm{~cm}^{-2}$ can still result in stable defects, known as boron-interstitial clusters. In summary, both dislocation loops and boron interstitial clusters that have been attributed to lifetime degradation have been revealed in the simulations under different implant and annealing conditions.

In the case of low dose boron implantation, the experimental $\mathrm{J}_{\mathrm{oe}}$ shows improvement with higher thermal budget, implying more complete defect dissolution. Although simulations do not reveal any stable, extended defects, these results can be attributed to deeply-penetrating defects which are shown to be detrimental to wafer bulk properties and device performance.

\section{ACKNOWLEDGMENTS}

The authors would like to thank the staff of the DIMES-ICP cleanroom for ion implantation and help with the annealing of the experimental samples; and Wim Tiwon for setting up the simulation environment.

\section{REFERENCES}

1. N. Bateman, P. Sullivan, C. Reichel, J. Benick, and M. Hermle , Energy Procedia 8, 509-514 (2011).

2. J. A. Minnucci, A. R. Kirkpatrick, and K. W. Matthei, IEEE Trans Electron Devices ED-27, 802-806 (1980).

3. R. T. Young, G. A. van der Leeden, R. L. Sandstrom, R. F. Wood, and R. D. Westbrook, Appl. Phys. Lett., 43, 666-668 (1983)

4. D. H. Macdonald, H. Maeckel, S. Doshi, W. Brendle, A. Cuevas, J. S. Williams and M. J. Conway, Appl. Phys. Lett. 82, 2987-2989 (2003).

5. D. H. Macdonald, P. N. K. Deenapanray and S. Diez, J. Appl. Phys. 96, 3687-3691 (2004).

6. B. J. Pawlak, T. Janssens, S. Singh, I. Kuzma-Filipek, J. Robbelein, N. E. Posthuma, J. Poortmans, F. Cristiano, and E. M. Bazizi, Prog. Photovolt: Res. Appl., (2011).

7. S. Prucnal, T. Shumann, W. Skorupa, B. Abendroth, K. Krockert and H. J. Moller, Acta Physica Polonica A, 120, 30-34 (2011).

8. R. A. Sinton and A. Cuevas, Appl. Phys. Lett. 69, 2510 (1996).

9. K. R. C. Mok, B. Colombeau, F. Benistant, R. S. Teo, S. H. Yeong, B. Yang, M. Jaraiz and S. Chu, IEEE Trans. Electron. Devices, 54, 2155-2163 (2007).

10. I. Martin-Bragado, I. Avci, N. Zographos, M. Jaraiz and P. Castrillo, Solid State Electron.52, 1430 (2008). 